\title{
Reasons for cancer metastasis: A holistic perspective (Review)
}

\author{
RUI-AN WANG ${ }^{1-3}$, YOU-YONG LU ${ }^{4}$ and DAI-MING FAN ${ }^{1,5}$ \\ ${ }^{1}$ State Key Laboratory of Cancer Biology; ${ }^{2}$ Department of Pathology, Xijing Hospital; ${ }^{3}$ Department of Pathology \\ and Pathophysiology, The Fourth Military Medical University, Xi'an, Shaanxi 710032; ${ }^{4}$ Laboratory of Molecular Oncology, \\ Key Laboratory of Carcinogenesis and Translational Research, Ministry of Education, Peking University Cancer Hospital \\ and Institute, Beijing 100142; ${ }^{5}$ Xijing Hospital of Digestive Diseases, The Fourth Military \\ Medical University, Xi'an, Shaanxi 710032, P.R. China
}

Received September 2, 2014; Accepted February 26, 2015

DOI: $10.3892 / \mathrm{mco} .2015 .623$

\begin{abstract}
Over several years, scientists investigating cancer have focused their efforts on elucidating the mechanisms underlying cancer metastasis, with the aim of finding a way to inhibit this process. These mechanisms, however, only explain the process of cancer metastasis, but do not explain why cancer would metastasize in the first place. Cancer metastasizes due to several factors, namely attack by the immune system, lack of oxygen and necessary nutrients, large amounts of lactic acid produced by glycolysis and increased cell death. Therefore, the majority of the presently available treatments for cancer also bear the potential to induce metastasis. Thus, it is crucial in medical practice to minimize the risk of cancer metastasis during a time when there are no effective means to inhibit this process.
\end{abstract}

\section{Contents}

1. Metastasis - predetermined or acquired genetic trait

2. Necrosis and apoptosis are associated with metastasis

3. Immune reaction/inflammation stimulates metastasis

4. Warburg effect and metastasis

5. Promotion of blood circulation vs. metastasis

6. Clinical implications

\section{Introduction}

Two paramount topics are central to the ongoing cancer research, namely the initiation and metastasis of cancer. The mechanism underlying cancer development remains

Correspondence to: Professor Rui-An Wang, Department of Pathology and Pathophysiology, The Fourth Military Medical University, 17 West Changle Road, Xi'an, Shaanxi 710032, P.R. China E-mail: wangra@fmmu.edu.cn

Key words: cancer, metastasis, necrosis, apoptosis, hypoxia, glycolysis, Warburg effect, radiotherapy, chemotherapy debated upon and several mechanisms have been suggested, including gene mutation (1), disorganization of tissue structure $(2,3)$, or genome and chromosome scrambling (4). As regards metastasis, there is also significant debate regarding whether metastasis is an inherited or acquired trait (5) and whether epithelial-to-mesenchymal transition is a prerequisite, an important first step for both invasion and metastasis (6), or merely an artificial phenomenon, i.e., a mirage (7). At the era of reductionism, scientists investigating cancer are focusing their efforts on elucidating the molecular mechanisms underlying the migration of cancer cells, revealing a complex process involving at least hundreds, if not thousands of molecules. The aim of this study was to address the issue of metastasis from a level beyond individual molecules, in order to determine the reason behind cancer cells moving out of their original location. We hypothesize that, by elucidating the molecular mechanisms cancer uses to metastasize, we may be able to design strategies to inhibit the process of metastasis. Alternatively, by understanding why cancer metastasizes, we may be able to alleviate the factors that induce migration of cancer cells.

\section{Metastasis - predetermined or acquired genetic trait}

The question as to why cancer metastasizes may appear simplistic for the average scientist, who may categorize this trait as a basal, innate instinct caused by genetic mutation. The prevailing theory of cancer is that it all begins with a single or even a series of genetic mutations. If metastasis is a trait of cancer, then it must also share the same origin. However, when considering cellular growth and development in the embryonic stage of any organism, a number of embryonic cells, such as primordial germ cells and neurocrest cells, naturally migrate over long distances to their final location, without any genetic mutations. Furthermore, evidence-based medicine requires evidence to support all theories. However, no single gene has yet been identified as responsible for metastasis, even after numerous genome-wide sequencing analyses.

Suzuki and Tarin (8) suggested that metastasis is an acquired trait, based on the significant differences in the gene expression profile between primary and lymph node metastatic breast cancer observed through microarrays. However, other researchers may rebut by pointing out that the 
metastasis originated in the primary site and not in the lymph node per se (9).

\section{Necrosis and apoptosis are associated with metastasis}

When investigating the reasons for cancer metastasis, we must begin with known facts and premises. Cancer cells are living, reproducing cells that are capable of movement if not bound or restricted by other cells or structures. Cancer cells possess a certain amount of autonomy after leaving their site of origin within the epithelium. However, although cancer cells are capable of movement, this ability is put to use when these cells require sustenance or when they must avoid danger.

Necrosis is crucial for the diagnosis of malignancy. In diagnostic pathology, the presence and extent of necrosis are important references for the diagnosis of malignancy. Although there is no proven explanation for the cause of necrosis, the most plausible explanation is that the tumor overgrows the ability of the circulatory system to supply sufficient nutrients. In fact, extensive necrosis is a common indicator of metastasis. For example, axillary lymph node metastasis was detected in a case of intracapsular carcinoma of the mammary gland. This type of lesion is usually considered as in situ carcinoma, which is rarely associated with metastasis. In fact, lymph node metastasis is not rare in the comedo type of ductal carcinoma in situ of the breast, which is characterized by central necrosis. The strong association of tumor necrosis with metastasis indicates that cell death per se or a factor closely asociated with cell death, such as lack of blood supply, is a strong stimulator of cancer metastasis (Fig. 1).

Increased apoptosis is associated with a higher grade of malignancy and poorer clinical outcome. Despite the widely accepted hypothesis that cancer cells are characterized by 'resistance to apoptosis' (10), malignant tumors display in fact an even higher occurrence of apoptosis compared with corresponding benign tumors or normal tissues. Pathological studies have repeatedly demonstrated that increased apoptosis is associated with a higher grade of malignancy and poor clinical outcome (11-15). Furthermore, overexpression of the anti-apoptotic protein Bcl-2 is an indicator of a favorable prognosis in breast cancer (16-20), colorectal cancer $(21,22)$ and non-small-cell lung cancer (NSCLC) (23). Conversely, overexpression of the cell death receptor CD95 is associated with poor clinical outcome in solid tumors, such as renal cell carcinoma and melanoma $(24,25)$.

The results described in the abovementioned studies appear to be contradictory. However, this may not be the case. In the biosphere, long lifespan and high fecundity are two mutually non-cooperative genetic traits. Organisms with short lifespans must correspondingly exhibit high fecundity (26). Otherwise, they would be considered unfit by Darwinian standards and find it difficult to propagate. Conversely, organisms with long lifespans must exhibit lower fecundity, otherwise they would dominate the biosphere and disrupt the balance of species. Cancer cells are autonomous cells that have control over their own lives. Therefore, increased cell death, either through necrosis or apoptosis, would stimulate the proliferation of surviving cells. Conversely, extending the lifespan of

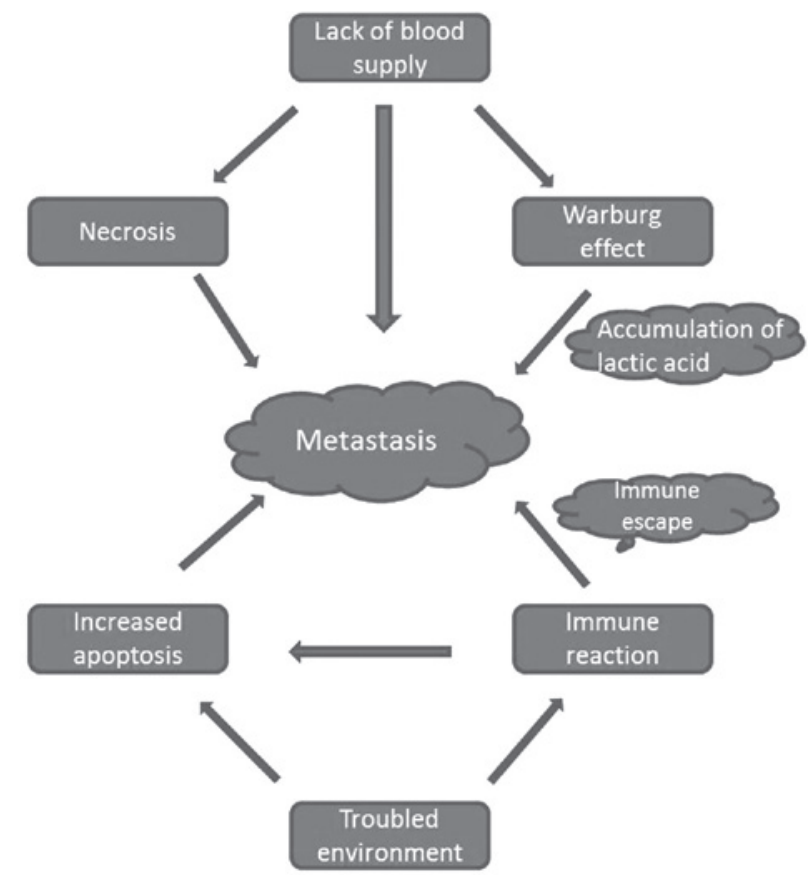

Figure 1. Factors promoting cancer cell metastasis. Troubled environment, including immune reactions, lack of blood supply leading to the Warburg effect, increased apoptosis and necrosis, drive cancer cells to migrate for survival.

cancer cells would inhibit cell growth, as demonstrated by the overexpression of Bcl-2 (27-29). Similarly, knockdown of apoptosis-promoting proteins also resulted in the inhibition of tumor growth, as in the case of CD95 (30), caspase-3 (31) and c-Jun N-terminal kinases (32).

The increased apoptosis associated with poor clinical outcome is not merely attributed to the fast growth of cancer. In fact, it appears reasonable to hypothesize that, under conditions of increased cell death, surviving cells are likely to move away (Fig. 1).

A recently published study reported solid evidence supporting this hypothesis. An inhibitor of the inhibitor of apoptosis protein, which was designed for the treatment of cancer through inducing apoptosis, was found to facilitate the metastasis of breast cancer cells to bone tissues (33). Should this hypothesis prove to be correct, a number of studies are expected to be published reporting similar findings, particularly since several drugs that induce apoptosis are currently in the stage of clinical trial.

\section{Immune reaction/inflammation stimulates metastasis}

The association between immune reaction and cancer is an interesting paradox. For several years, immunosurveillance has been considered an important barrier for carcinogenesis and a number of studies and clinical cares aim to prevent and treat cancer by enhancing the immune system. However, inflammation, which is an immune reaction, is widely accepted as a facilitator of carcinogenesis and cancer metastasis.

Although several studies have demonstrated that cytokines, such as interleukins and other cytokines released by immunocytes, are able to promote cancer cell proliferation (34), we 
hypothesized that the immune reaction against cancer cells per se is aimed at destroying these cells. However, the dead cells resulting from immune reactions may further stimulate cell proliferation and cancer metastasis (35). When under attack, the natural response of any organism is to defend itself or escape; cancer cells tend to escape (Fig. 1).

'Immune escape' has been described as a hallmark of cancer by Hanahan and Weinberg (10). In fact, escape from attack is a natural response in the biosphere rather than the patent of cancer cells. The immune escape techniques used by cancer cells include downregulation of the expression of major histocompatibility complex molecules, by which cancer cells try to become invisible to immune cells. The other important strategy is escape. It has been demonstrated that macrophages and other immunocytes promote cancer cell metastasis (36). Therefore, we agree with Prehn and Prehn (37) that immunosuppression may be a better approach to treating cancer compared with immunostimulation.

\section{Warburg effect and metastasis}

The predilection of cancer cells to engage in a high rate of glycolysis, even under conditions of adequate oxygen supply, is referred to as the Warburg effect and was first described by the famous German biochemist Otto Warburg in 1924 (38). Approximately 90 years after its discovery, the Warburg effect has again attracted significant attention in the field of cancer research (38). A number of researchers suggest that glycolysis renders cancer cells superior to their normal peers regarding proliferation (39). However, glycolysis produces large amounts of lactic acid, thereby significantly increasing the acidity of the surrounding environment. Therefore, cancer cells tend to move away from this hostile environment (Fig. 1). It was previously demonstrated that low local $\mathrm{pH}$ stimulated cancer invasion and metastasis (40); by neutralizing the acidic $\mathrm{pH}$, the occurrence of invasion and metastasis was reduced (41).

\section{Promotion of blood circulation vs. metastasis}

In traditional Chinese medicine, the cause of cancer was considered to be 'blood stasis'. Consequently, the guiding principle of cancer treatment in traditional medicine is to promote blood circulation. Over several decades, promoting blood circulation with Chinese medicine combined with radiotherapy has been used to enhance the efficacy of radiotherapy, based on the hypothesis that increased blood flow provides more oxygen to the tissues, which is critical for radiotherapy. Indeed, the efficacy of radiotherapy was significantly increased. However, the metastasis rate was also increased. Thus, clinical oncologists in China are quite resistant to the use of treatments aimed at promoting blood circulation, which is consistent with the concept that blood vessels provide pathways for cancer to metastasize.

It would appear that anti-angiogenesis may be used to starve cancer cells, as well as to inhibit their metastasis. Unexpectedly, however, anti-angiogenesis has also been found to stimulate cancer metastasis (42-44). It may appear puzzling that cancer cell metastasis may be stimulated by promoting blood circulation as well as by inhibiting angiogenesis. The explanation for this phenomenon lies with the fact that the promotion of blood circulation was not applied alone, but rather was used together with radiotherapy, which kills or injures cancer cells and forces them to metastasize. Promoting blood circulation provides the cells with the means to metastasize at the right time. If promotion of blood circulation was used alone, it would not have triggered cancer cell metastasis. Indeed, it was demonstrated that improving blood circulation reduced cancer metastasis (45). However, the problem with using Chinese medicine to promote blood circulation alone, is that it would be difficult to detect a tumor size reduction or disappearance; this may be difficult for physicians and patients to accept.

Regarding anti-angiogenesis, as mentioned above, blocking blood circulation would deprive cancer cells of their means for survival, which would naturally invoke a metastasis response. Furthermore, blocking angiogenesis would result in further apoptosis, invoking a higher degree of glycolysis and accumulation of lactic acid. All these phenomena, in turn, would stimulate cancer cell migration. Despite the lower availability of blood vessels, the cancer cells may invade further and use the lymphatics and blood vessels in the stroma to evade.

\section{Clinical implications}

Metastasis accounts for $>90 \%$ of cancer-related mortality. Therefore, reducing metastasis is the key to curtailing the rate of death from cancer. The quandary is that there is currently no method effective in blocking or impeding metastasis. By contrast, almost all available treatment approaches, including surgical resection, have the potential of stimulating the metastatic growth of cancer. Therefore, rather than investigating methods of eliminating cancer cells, we should be looking into methods for inhibiting cancer growth and metastasis. Instead of starving cancer cells by inhibiting angiogenesis, it may be preferable to 'feed' cancer cells by promoting blood circulation; and instead of inducing apoptosis of cancer cells by targeting the anti-apoptotic proteins, it may be preferable to prolong the lifespan of cancer cells through overexpression of these proteins, as living with cancer may be preferable to dying from cancer.

\section{Acknowledgements}

The authors would like to thank Jiasen Wang, Rice University, USA, for helping with the language improvement and William CS Cho, Queen Elizabeth Hospital, Hong Kong, for constructive comments and suggestions.

\section{References}

1. Vaux DL: In defense of the somatic mutation theory of cancer. Bioessays 33: 341-343, 2011.

2. Sonnenschein C and Soto AM: Somatic mutation theory of carcinogenesis: Why it should be dropped and replaced. Mol Carcinog 29: 205-211, 2000.

3. Soto AM and Sonnenschein C: The tissue organization field theory of cancer: a testable replacement for the somatic mutation theory. Bioessays 33: 332-340, 2011.

4. Duesberg P and Rasnick D: Aneuploidy, the somatic mutation that makes cancer a species of its own. Cell Motil Cytoskeleton 47: 81-107, 2000.

5. Zhang LJ, Li H, Zhang LY, et al: Metastasis: inherent vs. acquired phenotype. Med Hypotheses 74: 874-876, 2010. 
6. Weinberg RA: Mechanisms of malignant progression. Carcinogenesis 29: 1092-1095, 2008.

7. Tarin D, Thompson EW and Newgreen DF: The fallacy of epithelial mesenchymal transition in neoplasia. Cancer Res 65: 5996, 2005.

8. Suzuki M and Tarin D: Gene expression profiling of human lymph node metastases and matched primary breast carcinomas: clinical implications. Mol Oncol 1: 172-180, 2007.

9. Weinberg RA: Is metastasis predetermined? Mol Oncol 1: 263-264, 265-266, 2007.

10. Hanahan D and Weinberg RA: Hallmarks of cancer: the next generation. Cell 144: 646-674, 2011.

11. Lipponen P, Aaltomaa S, Kosma VM and Syrjanen K: Apoptosis in breast cancer as related to histopathological characteristics and prognosis. Eur J Cancer 30A: 2068-2073, 1994.

12. Lipponen PK and Aaltomaa S: Apoptosis in bladder cancer as related to standard prognostic factors and prognosis. J Pathol 173 333-339, 1994

13. Lipponen P: Apoptosis in breast cancer: relationship with other pathological parameters. Endocr Relat Cancer 6: 13-16, 1999.

14. Nishimura R, Nagao K, Miyayama H, et al: Apoptosis in breast cancer and its relationship to clinicopathological characteristics and prognosis. J Surg Oncol 71: 226-234, 1999.

15. Sinicrope FA, Hart J, Hsu HA, Lemoine M, Michelassi F and Stephens LC: Apoptotic and mitotic indices predict survival rates in lymph node-negative colon carcinomas. Clin Cancer Res 5: 1793-1804, 1999.

16. Lee KH, Im SA, Oh DY, et al: Prognostic significance of bcl-2 expression in stage III breast cancer patients who had received doxorubicin and cyclophosphamide followed by paclitaxel as adjuvant chemotherapy. BMC Cancer 7: 63, 2007.

17. Ali HR, Dawson SJ, Blows FM, et al: A Ki67/BCL2 index based on immunohistochemistry is highly prognostic in ER-positive breast cancer. J Pathol 226: 97-107, 2012.

18. Callagy GM, Pharoah PD, Pinder SE, et al: Bcl-2 is a prognostic marker in breast cancer independently of the Nottingham prognostic index. Clin Cancer Res 12: 2468-2475, 2006.

19. Callagy GM, Webber MJ, Pharoah PD and Caldas C: Meta-analysis confirms BCL2 is an independent prognostic marker in breast cancer. Bmc Cancer 8: 153, 2008.

20. Rolland P, Spendlove I, Madjd Z, et al: The 553 positive Bcl-2 negative phenotype is an independent marker of prognosis in breast cancer. Int J Cancer 120: 1311-1317, 2007.

21. Poincloux L, Durando X, Seitz JF, et al: Loss of Bcl-2 expression in colon cancer: a prognostic factor for recurrence in stage II colon cancer. Surg Oncol 18: 357-365, 2009.

22. Sinicrope FA, Hart J, Michelassi F and Lee JJ: Prognostic value of bcl-2 oncoprotein expression in stage II colon carcinoma. Clin Cancer Res 1: 1103-1110, 1995.

23. Tomita M, Matsuzaki Y, Edagawa M, Shimizu T, Hara M and Onitsuka T: Prognostic significance of bcl-2 expression in resected pN2 non-small cell lung cancer. Eur J Surg Oncol 29 654-657, 2003

24. Macher-Goeppinger S, Bermejo JL, Wagener N, et al: Expression and prognostic relevance of the death receptor CD95 (Fas/APO1) in renal cell carcinomas. Cancer Lett 301: 203-211, 2011.

25. Ugurel S, Rappl G, Tilgen W and Reinhold U: Increased soluble CD95 (sFas/CD95) serum level correlates with poor prognosis in melanoma patients. Clin Cancer Res 7: 1282-1286, 2001.
26. Partridge L, Gems D and Withers DJ: Sex and death: what is the connection? Cell 120: 461-472, 2005.

27. Knowlton K, Mancini M, Creason S, Morales C, Hockenbery D and Anderson BO: Bcl-2 slows in vitro breast cancer growth despite its antiapoptotic effect. J Surg Res 76: 22-26, 1998.

28. Pietenpol JA, Papadopoulos N, Markowitz S, Willson JK, Kinzler KW and Vogelstein B: Paradoxical inhibition of solid tumor cell growth by bcl2. Cancer Res 54: 3714-3717, 1994.

29. O'Reilly LA, Huang DC and Strasser A: The cell death inhibitor $\mathrm{Bcl}-2$ and its homologues influence control of cell cycle entry. Embo J 15: 6979-6990, 1996.

30. Chen L, Park SM, Tumanov AV, et al: CD95 promotes tumour growth. Nature 465: 492-496, 2010.

31. Huang Q, Li F, Liu X, et al: Caspase 3-mediated stimulation of tumor cell repopulation during cancer radiotherapy. Nat Med 17: 860-866, 2011

32. Hui L, Zatloukal K, Scheuch H, Stepniak E and Wagner EF: Proliferation of human HCC cells and chemically induced mouse liver cancers requires JNK1-dependent p21 downregulation. J Clin Invest 118: 3943-3953, 2008.

33. Yang C, Davis JL, Zeng R, et al: Antagonism of inhibitor of apoptosis proteins increases bone metastasis via unexpected osteoclast activation. Cancer Discov 3: 212-223, 2013.

34. Lippitz BE: Cytokine patterns in patients with cancer: a systematic review. Lancet Oncol 14: e218-e228, 2013.

35. Wang RA, Li QL, Li ZS, et al: Apoptosis drives cancer cells proliferate and metastasize. J Cell Mol Med 17: 205-211, 2013.

36. Fidler IJ: Macrophages and metastasis - a biological approach to cancer therapy. Cancer Res 45: 4714-4726, 1985.

37. Prehn RT and Prehn LM: Cancer immunotherapy by immunosuppression. Theor Biol Med Model 7: 45, 2010.

38. Thompson CB: Rethinking the regulation of cellular metabolism. Cold Spring Harb Symp Quant Biol 76: 23-29, 2011.

39. Bonuccelli G, Tsirigos A, Whitaker-Menezes D, et al: Ketones and lactate 'fuel' tumor growth and metastasis: Evidence that epithelial cancer cells use oxidative mitochondrial metabolism. Cell Cycle 9: 3506-3514, 2010.

40. Estrella V, Chen T, Lloyd M, et al: Acidity generated by the tumor microenvironment drives local invasion. Cancer Res 73: $1524-1535,2013$

41. Ibrahim HA, Cornnell HH, Coelho RML, et al: Reduction of metastasis using a non-volatile buffer. Clin Exp Metastasis 28: 841-849, 2011.

42. Ebos JM, Lee CR, Cruz-Munoz W, Bjarnason GA, Christensen JG and Kerbel RS: Accelerated metastasis after short-term treatment with a potent inhibitor of tumor angiogenesis. Cancer Cell 15: 232-239, 2009.

43. Paez-Ribes M, Allen E, Hudock J, et al: Antiangiogenic therapy elicits malignant progression of tumors to increased local invasion and distant metastasis. Cancer Cell 15: 220-231, 2009.

44. Zhu XD, Sun HC, Xu HX, et al: Antiangiogenic therapy promoted metastasis of hepatocellular carcinoma by suppressing host-derived interleukin-12b in mouse models. Angiogenesis 16 809-820, 2013

45. Wang WQ, Liu L, Sun HC, et al: Tanshinone IIA inhibits metastasis after palliative resection of hepatocellular carcinoma and prolongs survival in part via vascular normalization. J Hematol Oncol 5: 69, 2012. 